\title{
Multi-innovation Self-tuning Kalman Filter with Unknown Parameters Systems
}

\author{
Jun Yue ${ }^{1,2, a}$, Ying Shi ${ }^{1, b}$ \\ ${ }^{1}$ Electrical Engineering College, Heilongjiang University, Harbin, 150000, China \\ ${ }^{2}$ Chengdong College, Northeast Agricultural University, Harbin, 150000, China \\ aemail: yuejun0416@163.com, bemail: shiying89@hlju.edu.cn
}

Keywords: multi-innovation; Kalman filter; self-tuning filter; least squares

\begin{abstract}
Based on the multi-innovation least squares algorithm and the optimal Kalman filtering method, a new multi-innovation self-tuning Kalman filtering algorithm is presented for systems with unknown model parameters. It avoids the flaw of classical Kalman filter which needs to accurately know the model parameter in system. A simulation example shows its effectiveness.
\end{abstract}

\section{Introduction}

The existing information fusion Kalman filtering are mainly concentrated on systems with known model parameters and noise variances. However, in many applications, the model parameters and noise variances are fully or partially unknown. Systems with unknown model parameters and/or noise variance filter called self-tuning filter[1-3]. Self-tuning filtering principle is based on the autoregressive moving average (ARMA) innovation model of recursive identification, to get the estimation of unknown model parameters and noise variance, and then put the valuation generation into the known model parameters and noise variance of the optimal filter get self-tuning filter[4].So far, the research on self-tuning filter identification with the unknown system noise variance $\mathrm{Q}$ and $\mathrm{R}$ is more[5], while less recognition model parameters are unknown.

Multi-innovation identification[7] is an important branch of system identification. The innovation is the useful information that can improve parameter or state estimation accuracies. When the system has untrusted input data, the proposed algorithm can avoid the bad data and the loss of data on the influence of system parameter estimation, and a more accurate parameter estimation is obtained.

In this article, for systems with unknown model para-meters, through multi-innovation identification method of least squares, parameter values are estimated, then were put into the optimal Kalman filter. Then multi-innovation Kalman self-tuning filter for the system with unknown parameters was obtained.

\section{Problem Description}

Considering the linear time-invariant system

$$
\begin{aligned}
& x(t+1)=\Phi x(t)+\Gamma \omega(t) \\
& y(t)=H x(t)+v(t)
\end{aligned}
$$


Where $t$ is the discrete time, the state $x(t) \in R^{n}$, the measurement $y(t) \in R, \Phi=\left[\begin{array}{ccccc}0 & 1 & 0 & \cdots & 0 \\ 0 & 0 & 1 & \cdots & 0 \\ \vdots & \vdots & \vdots & & \vdots \\ 0 & 0 & 0 & \cdots & 1 \\ -a_{n} & -a_{n-1} & -a_{n-2} & \cdots & -a_{1}\end{array}\right], \Gamma=\left[\begin{array}{c}b_{1} \\ b_{2} \\ \vdots \\ b_{n}\end{array}\right]$ and $H=\left[\begin{array}{llll}c_{1} & c_{2} & \cdots & c_{n}\end{array}\right]$ are constant matrices.

Assumption1. $\omega(t)$ and $v(t)$ are independent white noises with zero means and variance matrices $\mathrm{Q}$ and $\mathrm{R}$, respectively.

Assumption2.The initial state $x(0)$ with mean $\mu_{0}$ and variance matrix $P_{0}$ is uncorrelated with $\omega(t)$ and $v(t)$.

Assumption3.The initial measurement time $t_{0}=1$.

When the model parameters $a_{1}, a_{2}, \cdots, a_{n}$ are unknown, calculate the Kalman state filter $\hat{x}(t \mid t)$.

\section{Kalman Filter}

When the model parameters $a_{1}, a_{2}, \cdots, a_{n}$ are known, the optimal Kalman filter

$$
\begin{aligned}
& \hat{x}(t+1 \mid t)=\Phi(t) \hat{x}(t \mid t) \\
& \varepsilon(t+1)=y(t+1)-H(t+1) \hat{x}(t+1 \mid t) \\
& P(t+1 \mid t)=\Phi(t) P(t \mid t) \Phi^{T}(t)+\Gamma(t) Q(t) \Gamma^{T}(t) \\
& K(t+1)=P(t+1 \mid t) H^{T}(t+1)\left[H(t+1) P(t+1 \mid t) H^{T}(t+1)+R(t+1)\right]^{-1} \\
& \hat{x}(t+1 \mid t+1)=\hat{x}(t+1 \mid t)+K(t+1) \times \varepsilon(t+1) \\
& P(t+1 \mid t+1)=\left[I_{n}-K(t+1) H(t+1)\right] \times P(t+1 \mid t)
\end{aligned}
$$

Where $I_{n}$ is the $\mathrm{n} \times \mathrm{n}$ unit matrix, $\hat{x}(0 \mid 0)=\mu_{0}, P(0 \mid 0)=P_{0}$.

The optimal Kalman filter method requires the system to have precise mathematical model and noise statistical noise mean and variance. When the model parameters are unknown, the state space model was changed into an ARMA model, which is used to identify the model parameters by least squares method.

Through(1), $x(t)$ can be represented as

$$
x(t)=\left(I_{n}-q^{-1} \Phi\right)^{-1} \Gamma \omega(t-1)
$$

Where $q^{-1}$ is the backward shift operator, substituing (9) into (2) yields

$$
y(t)=H\left(I_{n}-q^{-1} \Phi\right)^{-1} \Gamma \omega(t-1)+v(t)
$$

Applying the formula of matrix inverse,

$y(t)=\frac{\operatorname{Hadj}\left(I_{n}-q^{-1} \Phi\right) \Gamma}{\varphi\left(q^{-1}\right)} \omega(t-1)+v(t)$

From equation(11) we obtain the ARMA innovation model 


$$
\varphi\left(q^{-1}\right) y(t)=D\left(q^{-1}\right) \varepsilon(t)
$$

Where we define

$$
\begin{aligned}
& \varphi\left(q^{-1}\right)=\operatorname{det}\left(\mathrm{I}_{n}-q^{-1} \Phi\right) \\
& \varphi\left(\mathrm{q}^{-1}\right) y(t)=H \operatorname{adj}\left(\mathrm{I}_{n}-q^{-1} \Phi\right) \Gamma \omega(t-1)+v(t) \\
& H \operatorname{adj}\left(\mathrm{I}_{n}-q^{-1} \Phi\right) \Gamma \omega(t-1)+v(t)=D\left(q^{-1}\right) \varepsilon(t)
\end{aligned}
$$

\section{Multi-innovation Least Squares}

Considering the scalar system

$$
y(t)=\varphi^{T}(t) \theta+v(t)
$$

Where $y(t)$ is an output, $\varphi(t)$ is a vector of the input and output data, $\theta$ is a model parameter vector. $v(t)$ is a random noise with zero mean.

Based on the least squares identification algorithm to estimate (16) parameter vector $\theta$

$$
\hat{\theta}(t)=\hat{\theta}(t-1)+L(t) e(t)
$$

Where $L(t)$ is a gain vector, $e(t):=y(t)-\varphi^{T}(t) \hat{\theta}(t-1)$ is a scalar innovation.

Multi-innovation identification is the identification algorithm of the scalar innovation $(e(t) \in R)$ promotion for the innovation vector $\left(E(p, t) \in R^{p}\right)$,

$$
E(p, t)=\left[\begin{array}{c}
e(t) \\
e(t-1) \\
\vdots \\
e(t-p+1)
\end{array}\right]
$$

i.e. Multi-innovation least squares identification algorithm is got by modeling on the derivation process of the recursive least squares identification method, can get multi-innovation.

$$
\begin{aligned}
& \hat{\theta}(t)=\hat{\theta}(t-1)+L(t)\left[Y(p, t)-\Phi^{T}(p, t) \times \hat{\theta}(t-1)\right] \\
& L(t)=P(t) \Phi(p, t)=P(t-1) \Phi(p, t)\left[I_{p}+\Phi^{T}(p, t) P(t-1) \Phi(p, t)\right]^{-1} \\
& P(t)=P(t-1)-L(t) \Phi^{T}(p, t) P(t-1) \\
& \Phi(p, t)=[\varphi(t), \varphi(t-1), \cdots, \varphi(t-p+1)] \\
& Y(p, t)=[y(t), y(t-1), \cdots, y(t-p+1)]^{T} \\
& \text { Where } p \text { is the innovation length. }
\end{aligned}
$$

\section{Self-tuning Kalman Filter}

When the model parameters are unknown, the optimal Klaman filter is converted into the self-tuning Kalman filter by using the estimate of the model parameters. The procedure includes two steps:

Step 1 , the valuation $\hat{\theta}(t)$ of unknown parameters $\theta$ can be achieved by using multi-innovation least square identification method.

Step 2, the self-tuning Kalman filter is obtained with the estimate $\hat{\theta}(t)$ in the optimal Kalman filter. 


\section{Simulation Results}

Considering the linear time-invariant system

$$
\begin{aligned}
& x(t+1)=\Phi x(t)+\Gamma \omega(t) \\
& y(t)=H x(t)+v(t)
\end{aligned}
$$

Where $t$ is the discrete time, the state $x(t) \in R^{3}$, the measurement $y(t) \in R, \Phi=\left[\begin{array}{ccc}0 & 1 & 0 \\ 0 & 0 & 1 \\ -a_{3} & -a_{2} & -a_{1}\end{array}\right], \Gamma=\left[\begin{array}{l}0 \\ 0 \\ 1\end{array}\right]$ and $H=\left[\begin{array}{lll}1 & 0 & 0\end{array}\right]$ are constant matrices.

Taking $p=2$.

Where we define

$$
\begin{aligned}
& \theta:=\left[a_{1}, a_{2}, a_{3}\right]^{T} \\
& \varphi(t):=[-\mathrm{y}(\mathrm{t}-1),-\mathrm{y}(\mathrm{t}-2),-\mathrm{y}(\mathrm{t}-3), \mathrm{e}(\mathrm{t}-1), \mathrm{e}(\mathrm{t}-2), \mathrm{e}(\mathrm{t}-3)]^{T}
\end{aligned}
$$

Let $a_{1}=-0.7, a_{2}=-0.53, a_{3}=0.315, \mathrm{Q}=0.25, \mathrm{R}=0.16$. We obtain the valuation of unknown model parameters and Kalman filter. The simulation results are shown in Fig.1-3, where the liners stands for the real value $a_{1}, a_{2}, a_{3}$ and the curves are the estimates $\hat{a}_{1}, \hat{a}_{2}, \hat{a}_{3}$. The simulation results are shown in Fig.4-6, including the state $x_{1}, x_{2}, x_{3}$ and their estimates $\hat{x}_{1}, \hat{x}_{2}, \hat{x}_{3}$.

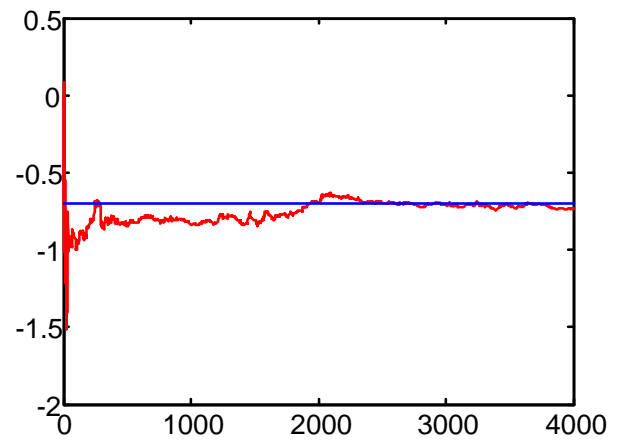

Fig. 1 The estimate $\hat{a}_{1}$ of $a_{1}$

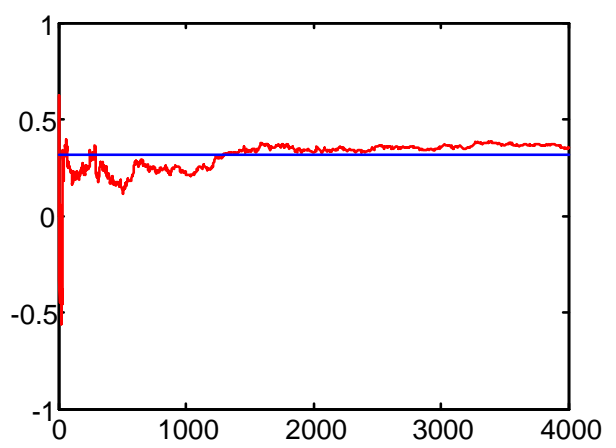

Fig.3 The estimate $\hat{a}_{3}$ of $a_{3}$

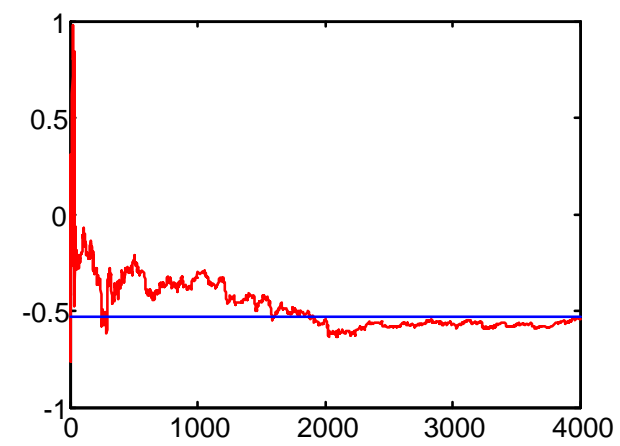

Fig.2 The estimate $\hat{a}_{2}$ of $a_{2}$

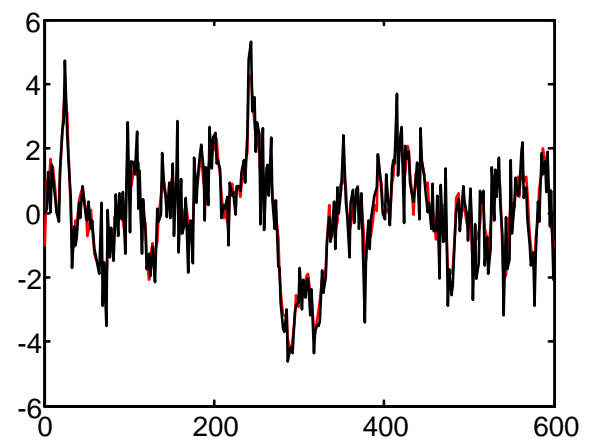

Fig.4 The state $\hat{x}_{1}$ of $x_{1}$ 


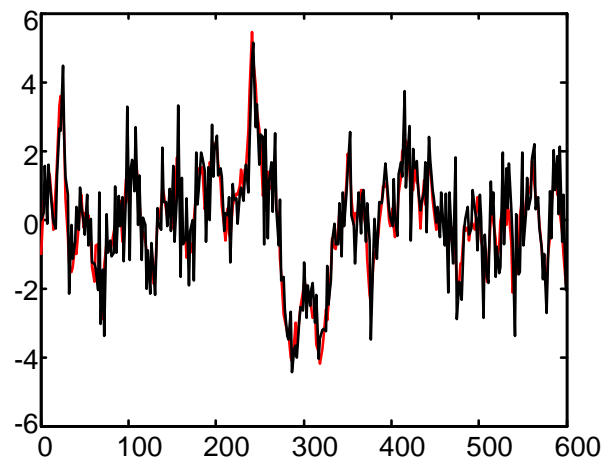

Fig.5 The state $\hat{x}_{2}$ of $x_{2}$

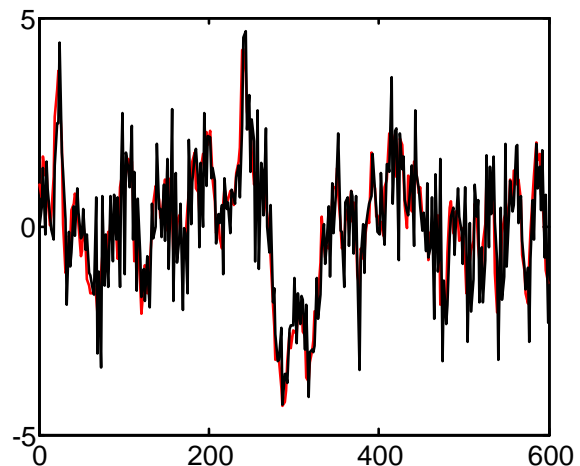

Fig. 6 The state $\hat{x}_{3}$ of $x_{3}$

\section{Conclusion}

For systems with unknown model parameters, through multi-innovation least-squares identification method, the valuation of the parameters are gained. Based on valuation of model parameters multi-innovation least-squares self-tuning Kalman filter is proposed. It has higher accuracy than that of the least-squares identification method. But the least-squares algorithm convergence speed, the efficiency of using the data itself is very high, so multi-innovation least-squares algorithm for parameter estimation accuracy improvement is very limited. Only if the data missing happens, recursive intervai-varying multi-innovation least-squares show more efficiency[7].

\section{Acknowledgement}

This article is supported by the National natural science fund project. Item No.61203121.

\section{References}

[1] Hagander P,Wittenmark B.A self-tuning filter for fixed-lag smoothing.IEEE Trans Information Theory,1977:23( 3) :377-384

[2] Fung P T K,Grimble M J.Dynamic ship positioning using a self-tuning Kalman filter.IEEE Tran Automatic Control,1983:( 28 ):339-350

[3] Moir T,Grimble M J.Optimal self-tuning filtering prediction and smoothering for discrete multivariable processes. IEEE Trans,Auto-matic Control,1984:( 29):128-137

[4] Deng Z L,Gao Y,Li C B,et al.Self-tuning decoupled informationfusion Wiener state component filters and their convergen-ce.Automatic,2008: (44):685-695

[5] Deng Z L,Li C B.Self-tuning information fusion Kalman predictor-weighted by diagonal matrices and its convergence anaysis.Acta Automatic Sinica,2007:33( 2) : 156-163

[6] DING Feng.System identification.Part F: Multi-innovation identification theory and methods.Journal of Nanjing University of Information Science and Technology: Natural Science Edition,2012,4( 1) : 1-28

[7] DING Feng.System identification theory and methods[M].Beijing: China: Electric Power Press, 2012:248-290

[8] LI Jun,WANG Xue-ping,ZHANG Fu-bin. Information Fusion based on the Self-Tuning Kalman Filter. Fire Control and Command Control, Vol.32,No.3March,2007

[9] Deng Z L,Wang X,Gao Y,Modeling and estimation[M]. Beijing: China :Science press, 2007:53-55 